\title{
Consonant discrimination by seven year olds: A pilot study
}

RONALD S. TIKOFSKY AND JAMES R. MCINISH UNIVERSITY OF MICHIGAN

Speech discrimination by four seven-year olds was tested on three consecutive days using a forced choice procedure. Ss determined whether pairs of initial consonants were the same or different. Degree of contrast between initial consonants ranged from zero to five distinctive features. Pairs differing by one distinctive feature yield the most errors. Some distinctive features contribute more to discriminability than others, and within a feature category some pairs are more discriminable than others.

Research on speech development has emphasized acquisition of articulatory proficiency while almost completely neglecting the problem of acquisition of speech discrimination. Jakobson \& Halle (1956) present a developmental description of speech discrimination acquisition based on distinctive features. They state that acquisition follows a sequence of successive binary contrasts going from those which are highly discriminable to minimal differences. Validity of the distinctive feature concept in speech discrimination has been demonstrated with adult subjects (Miller \& Nicely, 1955; Wickelgren, 1966). Observational reports summarized by Ervin \& Miller (1963) lend support to the utility of the distinctive features for a description of speech discrimination acquisition. Shvachkin's (1958) results support Jakobson's prediction of the ontogenetic sequence of discrimination ability. Shvachkin found that the voiced-voiceless discrimination is acquired late by Russian-speaking children. He attributes this to the "acoustic similarity between contrasting members of a pair and especially because of their articulatory identity."

Studies (Templin, 1957; Poole, 1938) show that by age 7 , most children articulate accurately all the phonemes of their native language, and demonstrate the sequential development of articulatory skill. They also suggest that by age 7 , most children can discriminate among the phonemes. The ability to discriminate between phonemes must therefore precede, or coincide with the acquisition of articulatory control.

As a precursor to a developmental study of speech discrimination acquisition based on distinctive features, the speech discrimination abilities of four 7-yearolds was tested using the Miller \& Nicely (1955) distinctive feature categories. Their classification system seems acceptable to Jakobson (1956, p. 27) as a means of identifying distinctive features on a perceptual basis.

It was hypothesized that: (1) by age 7 , Ss (because of their articulatory proficiency) would make low discrimination error scores; (2) as the number of differ- ences in terms of distinctive features increased, the lower the error rate; (3) some distinctive features contribute more to discriminability than others; and (4) within a distinctive feature category, some distinctions are more important than others.

\section{Nethod}

Three male and one female 7-year-old served as Ss. Ss were tested individually on a forced choice speech discrimination task for three consecutive days.

Stoudt's (1964) 120 item word-word (WW) wordnonsense syllable (WN), and nonsense syllable-nonsense syllable (NN) lists based on the Miller-Nicely (MN) features (1955) served as the stimuli. Lists were constructed using 15 different initial consonants. The resulting 105 different contrasting pairs include all possible pairings that could occur in English for three different lists for each of the WW, WN, and NN categories. Each consonant was paired with itself to yield 15 different noncontrasting pairs, which were randomly inserted into each stimulus list. Contrasting pairs differed by one to five $\mathrm{MN}$ features. Stuodt's practice pairs were used to acquaint $S$ with the task.

Stimuli were recorded on one channel of an Ampex 351-2 tape recorder, with a pulse on the second channel at the end of the speech signal of the second member of the stimulus pair.

Three lists, one of each type, were presented during a test session, with different sets of lists for each day. Order of presentation was the same each day. Practice pairs preceded list presentation.

Stimuli were played to $S$ through an Ampex 622 loudspeaker in a quiet room with $S$ seated before a response panel and in front of the speaker. At the end of the speech signal of the second member of the pair, the pulse stopped the tape recorder. The panel had a start point and two push buttons equidistant from it. Pushing a button caused it to light up. E reactivated the recorder by a foot switch. Ss were instructed to respond by pushing a button labelled "same" if they thought they heard both members of the pair as identical, or that marked "different" if they thought both members of the pair did not sound identical, and to respond as quickly as possible after listening to the pair. Ss were given no knowledge of results during the experiments.

Results

Only $2 \%(84)$ of the total number of responses (4320) made by all Ss for all lists and days were in error. This result offers strong support for the proposition 
that by age 7, children can discriminate accurately between initial consonant contrasts.

Despite the low error rate, most of the errors (70 out of 864 possible correct responses) occur for contrasts having a one MN feature difference. These errors account for $83 \%$ of all incorrect responses. Errors occur for only two other categories. Ss make 10 errors out of their 540 responses to noncontrasting pairs, and four errors out of their 1368 responses to pairs differing by two MN features. Thus it may be concluded that as the number of feature differences increases, error rate will drop. In fact, any increase beyond the one feature difference assures almost perfect discriminability in terms of this experiment.

Only two of the five features (place of articulation and voicing) used to obtain the MN one feature difference pairs generated error responses. Ss made 55 errors of 360 responses when pairs differed by place of articulation and 12 errors of 216 responses when the differentiating feature was voicing. This result supports the hypothesis that some features contribute more to ease of discriminability than others.

Two contrasting pairs for the place of articulation feature $/ f, \theta /$ and $/ \nabla, \delta /$ yield high error scores. Of the 36 responses made to each of these pairs, Ss make 25 errors for the $/ f, \theta /$ contrast and 26 errors for $/ v, \delta /$. These are the contrasts missed most often by Stoudt's (1964) aphasic and normal Ss, Shvachkin's children, and Miller \& Nicely's (1955) Ss. Of the six pairs with voicing as the contrasting feature, only three, $/ v, f /, / z, s /$ and $/ \delta, \theta /$ produced errors $(4,5$, and 3 errors respectively for each pair with 36 responses per contrasting pair). Error distribution for both voicing and place of articulation is nonrandom $\left(\chi^{2}, p<.01\right)$. Thus, the hypothesis that some pairings within a distinctive feature category are more important than others in assessing discriminability is supported.

Errors in response to noncontrasting pairs showed no discernible pattern. This result is confirmed by the Chi-square analysis.

The distribution of errors discussed above was present in the protocols of each subject. Thus, it is not possible to attribute the results to the performance pattern generated by only one of two Ss. A Chi-square analysis of the data reveals no significant $\mathrm{Ss}$, list or days effects.

\section{Discussion}

Results of this experiment support the contention that children by age 7 discriminate among the consonant phonemes of their native language, except for a small group of contrasting consonants which are not easily discriminable even for adults. Teachers of English as a foreign language and linguists have indicated to us that contrasts yielding the greatest number of errors in this study also pose the greatest discrimination problems for non-native speakers of English.

Three factors emerge as having an important bearing on ease of discrimination: number of feature differences; the particular feature; the contrasting pair of consonants. One-feature differences involving place of articulation or voicing generate the highest error scores. Distinctive feature systems offered by Jakobson or by Miller and Nicely do not deal adequately with the differential contribution made by these factors to the discrimination task.

\section{References}

ERVIN, SUSAN, \& MILLER, W. R. Language Development. Child Psychology. N.S.S.E. Yearbook, 1963. Harold Stevenson (Ed.), University of Chicago Press, Chicago, 1963.

JAKOBSON, R., \& HALLE, M. Fundamentals of Language. 'S-Gravenhage: Mouton, 1956.

MILLER, G. A., \& NICELY, PATRICIA. An Analysis of Perceptual Confusions Among Some English Consonants. J. Acous. Soc. Amer.,1952, 27, 338-352.

POOLE, IRENE. The genetic development of the articulation of consonant sounds. Unpublished Doctoral Dissertation. University of Michigan, 1934.

SHVACHKIN, N. H., Development of phonemic speech perception in early childhood. Abs. reported by Slobin, D. L. In The Genesis of Language. Smith, F., \& Miller, G. A. (Eds.), M.I.T. Press. Cambridge, 1966.

STOUDT, JR., R. J. A study of consonant discrimination by aphasics. Doctoral Dissertation, University of Michigan. Ann Arbor, Michigan: University Microfilms, 1964, No. 6003.

TEMPLIN, MILDRED. Certain Language Skills in Children: Their Development and Interrelationships. Minneapolis, University of Minnesota Press, 1957.

WICKELGREN, W. A. Distinctive features and errors in short-term memory for English consonants. J. Acous. Soc. Amer., 1966, 39, 388-398.

Notes

1. This research was supported by a grant from the National Institute for Child Health and Human Development, Grant Number 5 PO1 HDO 01368 .

2. This report is based on a paper presented at the meetings of the Midwestern Psychological Association, May, 1967, Chicago, Illinois. 